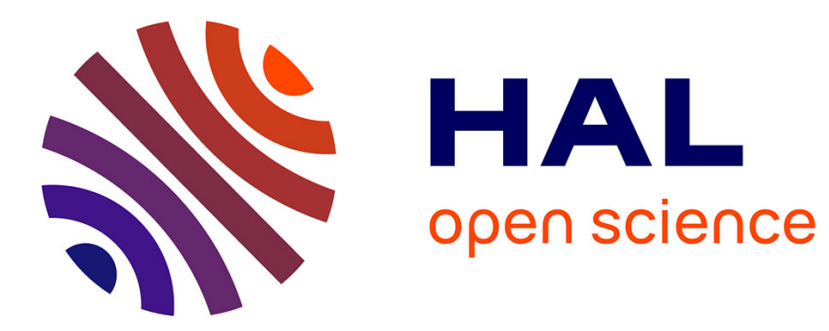

\title{
Modifications de propagation dues aux retards aléatoires
}

B. Lacaze

\section{To cite this version:}

B. Lacaze. Modifications de propagation dues aux retards aléatoires. Journal de Physique IV Proceedings, 1994, 04 (C5), pp.C5-1343-C5-1346. 10.1051/jp4:19945299 . jpa-00253012

\section{HAL Id: jpa-00253012 https://hal.science/jpa-00253012}

Submitted on 1 Jan 1994

HAL is a multi-disciplinary open access archive for the deposit and dissemination of scientific research documents, whether they are published or not. The documents may come from teaching and research institutions in France or abroad, or from public or private research centers.
L'archive ouverte pluridisciplinaire HAL, est destinée au dépôt et à la diffusion de documents scientifiques de niveau recherche, publiés ou non, émanant des établissements d'enseignement et de recherche français ou étrangers, des laboratoires publics ou privés. 


\title{
Modifications de propagation dues aux retards aléatoires
}

\author{
B. LACAZE
}

ENSEEIHT/LEN7/GAPSE, 2 rue Camichel, 31071 Toulouse, France

\begin{abstract}
Acoustic waves can undergo random delays because of propagation atmosphere turbulence. If the emitted wave is monochromatic with a frequency $f_{0}=\frac{\varpi_{0}}{2 \pi}$, the wave received at a point can be modelized in the form $U(t)=e^{i \varpi_{0}(t-A(t))}$ where $A(t)$ is the propagation delay. It is shown that, in this model, the received monochromatic wave has to be accompanied by a noise the power spectrum of which will depend on propagation delay variation speed. More particularly, the case where $A(t)$ is a gaussian process is examined and it is shown that this hypothesis is particularly appropriate to account for gas and liquid losses.
\end{abstract}

\section{L'onde monochromatique retardée aléatoirement}

Considérons l'onde monochromatique $e^{i \varpi_{0} t}$ de fréquence $f_{0}=\frac{\varpi_{0}}{2 \pi}$. Imaginons qu'elle se propage dans un certain milieu où la vitesse de propagation fluctue en fonction du temps pour diverses raisons : changements de trajectoires, variation des conditions macroscopiques ou de la composition du milieu, déplacement de l'émetteur ou du récepteur... Dans ces conditions, l'onde reçue s'écrit : $U(t)=e^{i \varpi_{0}(t-A(t))}$ où $A(t)$ est le retard de propagation. On le supposera dans la suite stationnaire au sens suivant :

$$
\begin{aligned}
& E\left[e^{-i \varpi A(t)}\right]=\Psi(\varpi) \\
& E\left[e^{-i \varpi(A(t)-A(t-\tau))}\right]=\Phi(\tau, \varpi)
\end{aligned}
$$

$\Phi$ et $\Psi$ sont donc des fonctions indépendantes de $t$. Il est équivalent de supposer que les lois de probabilité des variables aléatoires $A(t)$ et $A(t)-A(t-\tau)$ sont indépendantes de $t$. A l'aide de (1), on peut écrire :

$$
\begin{aligned}
& E[U(t)]=e^{i \varpi_{0} t} \Psi\left(\varpi_{0}\right) \\
& E\left[U(t) U^{*}(t-\tau)\right]=e^{i \varpi_{0} \tau} \Phi\left(\tau, \varpi_{0}\right)
\end{aligned}
$$

Posons :

$$
\begin{aligned}
& g(t)=e^{i \varpi_{0} t} \Psi\left(\varpi_{0}\right) \\
& V(t)=U(t)-g(t)
\end{aligned}
$$


On obtient immédiatement :

$$
\begin{aligned}
& E[V(t)]=0 \\
& E\left[V(t) V^{*}(t-\tau)\right]=e^{i \varpi_{0} \tau}\left(\Phi\left(\tau, \varpi_{0}\right)-\left|\Psi\left(\varpi_{0}\right)\right|^{2}\right)
\end{aligned}
$$

$V=\{V(t), t \in \mathcal{R}\}$ constitue donc une fonction aléatoire stationnaire (au sens large), puisque la variable $t$ a disparu dans les expressions précédentes.

Le théorème de Bochner-Khintchine[1] associe à la fonction d'autocorrélation de $V$, un spectre de puissance $S_{V}(\varpi)$ défini par :

$$
E\left[V(t) V^{*}(t-\tau)\right]=\int_{-\infty}^{\infty} e^{i \varpi \tau} d S_{V}(\varpi)
$$

Supposons maintenant que :

$$
\lim _{\tau \longrightarrow \infty} \Phi(\tau, \varpi)=|\Psi(\varpi)|^{2}
$$

(5) exprime l'indépendance (asymptotique) de $A(t)$ et $A(t-\tau)$ lorsque $\tau$ est grand. Cette condition est vérifiée dans tout milieu que l'on peut qualifier de turbulent. A cette condition, (3) :

$$
\lim _{\tau \rightarrow \infty} E\left[V(t) V^{*}(t-\tau)\right]=0
$$

Il est alors bien connu [2] que le spectre de puissance $S_{V}(\varpi)$ est continu. Sauf cas exceptionnel, il lui correspond une densité spectrale de puissance $s_{V}(\varpi)$ donnée d'après (3) et (4) par une transformée de Fourier inverse :

$$
s_{V}(\varpi)=\frac{d S_{V}(\varpi)}{d \varpi}=\frac{1}{2 \pi} \int_{-\infty}^{\infty} e^{i\left(\varpi_{0}-\varpi\right) \tau}\left(\Phi\left(\tau, \varpi_{0}\right)-\left|\Psi\left(\varpi_{0}\right)\right|^{2}\right) d \tau
$$

On voit donc que le processus reçu $U(t)=g(t)+V(t)$ est formé d'une partie monochromatique (déterministe) ajoutée à un bruit stationnaire de moyenne nulle et de spectre de puissance à densité. La partie monochromatique de fréquence $\frac{\varpi_{0}}{2 \pi}$ est le signal émis multiplié par la fonction caractéristique de $-A(t)$ prise au point $\varpi_{0} ;\left|\Psi\left(\varpi_{0}\right)\right|$ représente l'affaiblissement (en amplitude) de l'onde et le changement de phase de l'onde est en rapport direct avec l'argument de $\Psi\left(\varpi_{0}\right)$.

\section{L'hypothèse gaussienne}

Supposons que $A(t)$ est l'addition d'un grand nombre de retards élémentaires peu liés et d'importances comparables. On se place ainsi dans le cadre des hypothèses des théorèmes de la limite centrale et de la tendance vers la loi normale [3], [4]. Dans ce cas, on peut admettre que la fonction aléatoire $A=\{A(t), t \in \mathcal{R}\}$ est un processus gaussien stationnaire, alors complètement caractérisé par les fonctions caractéristiques [4] :

$$
\left\{\begin{array}{l}
\Psi(\varpi)=e^{-i m \varpi-\frac{\sigma^{2} \varpi^{2}}{2}} \\
\Phi(\tau, \varpi)=e^{-\sigma^{2} \varpi^{2}(1-\rho(\tau))}
\end{array}\right.
$$

où

$$
\begin{aligned}
m & =E\{A(t)\} \\
\sigma^{2} & =\operatorname{Var} A(t) \\
\sigma^{2} \rho(\tau) & =\operatorname{Cov}\{A(t), A(t-\tau)\}
\end{aligned}
$$

$\rho(\tau)$ est le coefficient de corrélation de $(A(t), A(t-\tau))$. Un modèle simple de liaison entre $A(t)$ et $A(t-\tau)$ est représenté par :

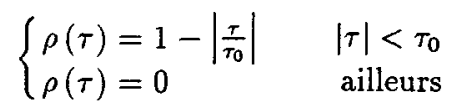


$\tau_{0}$ est le "rayon de corrélation" de $A(t)$. Un calcul élémentaire montre que, dans ce cas, $(6)$ s'écrit :

$$
\begin{aligned}
& s_{V}(\varpi)=\tau_{0} f\left[\tau_{0}\left(\varpi-\varpi_{0}\right)\right] \\
& f(u)=\frac{\gamma}{\pi\left(u^{2}+\gamma^{2}\right)}\left[1-e^{-\gamma} \cos u-\gamma e^{-\gamma \frac{\sin u}{u}}\right] \quad \gamma=\sigma^{2} \varpi_{0}^{2}
\end{aligned}
$$

La figure 1 représente $f /\left(1-e^{-\gamma}\right)$ lorsque la variable $u$ décrit l'intervalle $[0,20]$, le paramètre $\gamma$ prenant ses valeurs entre 0,01 et 15 , La figure 2 représente la puissance relative de $V(t)$ dans la bande $\left[\varpi_{0}-\frac{u}{\tau_{0}}, \varpi_{0}+\frac{u}{\tau_{0}}\right]$ c'est-à-dire $h(u)$ définie par :

$$
h(u)=\frac{1}{1-e^{-\gamma}}\left[S_{V}\left(\varpi_{0}+\frac{u}{\tau_{0}}\right)-S_{V}\left(\varpi_{0}-\frac{u}{\tau_{0}}\right)\right]=\frac{2}{1-e^{-\gamma}} \int_{0}^{u} f(x) d x
$$

pour $\gamma$ allant de 0,01 à 10 , c'est-à-dire pour des affaiblissements allant de 0,99 à 4,5.10-5.

Comme il était prévisible, le rayon de corrélation de retard représenté par le paramètre $\tau_{0}$ est un paramètre très important. Plus $\tau_{0}$ sera petit, et plus le spectre s'étalera (à puissance totale constante). Par exemple, pour $\varpi_{0}=10^{3}, \gamma=0,1, \tau_{0}=10^{-n}$, seulement $10 \%$ de la puissance de $V(t)$ se trouve dans un intervalle autour de $\varpi_{0}$ égal à $0,3.10^{n}$, soit, par exemple, $0,3 M H$ pour $n=6$ mais seulement $0,3 H$ pour $n=0$.

\section{Application à la transmission des ondes acoustiques}

1. Une onde se réfléchissant sur une surface mobile subit un retard fluctuant $A(t)$. C'est le cas, par exemple, de la surface de la mer. En général, le rayon de corrélation $\tau_{0}$ du retard sera grand vis-à-vis de la période de l'onde. A cette condition, le spectre de puissance de $V(t)$ sera concentré autour de la fréquence de l'onde. Et, donc, tout se passera comme si le spectre de l'onde reçue était élargi. Et cela, d'autant plus que la variance de $A(t)$ est grande $\left(\left|\Psi\left(\varpi_{0}\right)\right|\right.$ est plus petit) et que $\tau_{0}$ est plus petit.

2. Que ce soit dans un liquide ou un gaz, une onde de fréquence $f_{0}=\frac{\varpi_{0}}{2 \pi}$ subit un affaiblissement (en amplitude) représenté en première approximation par la loi [5] et [6] :

$$
a_{x}=a_{0} e^{-\alpha \varpi_{0}^{2} x}
$$

où $a_{0}$ et $a_{x}$ sont les amplitudes et $x$ la longueur du parcours ; l'affaiblissement "géométrique" dû à la surface des capteurs n'est pas pris en compte.

Quelles que soient les causes réelles de cet affaiblissement, les retards de propagation gaussiens permettent d'expliquer (11). Dans ce cas, en effet, l'affaiblissement de l'onde n'est autre que le module de $\Psi\left(\varpi_{0}\right)$, fonction caractéristique de $-A(t)$ prise en $\varpi_{0}$.

D'après (7), on a donc :

$$
\left\{\begin{array}{l}
m=\frac{x}{c} \\
\sigma^{2}=2 \alpha x
\end{array}\right.
$$

où $c$ est la célérité du son dans le fluide. En première approximation, on doit pouvoir admettre que $\rho(\tau)$ est indépendant de $x$ ( $\rho$ est le coefficient de corrélation du couple $(A(t), A(t+\tau)$ ). La constante $\alpha$ est donnée par des tables ou par la formule $\alpha=\frac{2 \eta}{3 \mu c^{3}}$ dans les gaz [7] ( $\eta$ : coefficient de viscosité, $\mu$ : densité). Dans l'air aux conditions normales, on a approximativement $\alpha=$ $3.10^{-15} \mathrm{~s}^{2} . \mathrm{cm}^{-1}$. Donc, pour $x=10^{3} \mathrm{~cm}, m=0,03 \mathrm{~s}$ et $\sigma=2,4.10^{-6} \mathrm{~s}$ ( $m$ est linéaire en $x$ et $\sigma$ en $\sqrt{x}$ ). On voit que des variations très faibles de $A(t)$ expliquent les affaiblissements réels. 
3. Dans le modèle étudié, l'onde monochromatique s'accompagne d'un bruit $V(t)$ dont le spectre de puissance est à densité $s_{V}(\varpi)$. Cette dernière quantité dépend essentiellement du "rayon de corrélation" $\tau_{0}$ du retard $A(t)$. Si les variations de $A(t)$ sont attribuées à la nature moléculaire du milieu (vitesse de déplacement et longueur des parcours entre chocs aléatoires), $\tau_{0}$ est très faible sans que l'on puisse prédire son ordre de grandeur. La nature du retard est dans ce cas fondamentalement différente de ce qu'elle était dans le paragraphe 1.

\section{Conclusion}

Les retards de propagation aléatoires sont cause d'affaiblissement des ondes monochromatiques. L'énergie perdue se retrouve sous la forme d'un bruit $V(t)$ dont la densité spectrale $s_{V}(\varpi)$ dépend, pour sa forme, essentiellement du rayon de corrélation $\tau_{0}$ du retard. Dans le cas où $\tau_{0}$ est très faible, $s_{V}(\varpi)$ sera largement étalé autour de la fréquence de l'onde émise. Dans le cas contraire ( $\tau_{0}$ très grand), le retard de propagation apparaitra comme un élargissement de la raie spectrale. Les retards gaussiens permettent d'autre part d'expliquer les affaiblissements de la forme $e^{-\alpha \varpi^{2} x}$ que l'on trouve particulièrement dans le cas des liquides et des gaz.

\section{References}

[1] Lamperti J., Stochastic Processes (Springer-Verlag, 1977) p43.

[2] Lukacs E., Characteristic Functions (Griffin, London, 1960) p20.

[3] Renyi A., Calcul des Probabilités (Dunod, Paris, 1966) p413.

[4] Gikhman I.I. and Skorokhod A.V., Introduction to the Theory of Random Processes (W.B.Saunders Company, London, 1965) p18.

[5] Landau L. et Lifchitz E., Mécanique des Fluides (MIR, Moscou, 1971) p379.

[6] Nielsen R.O., Sonar Signal Processing (Artech House, 1991) p94.

[7] Etter P.C., Underwater Acoustic Modelling (Elsevier, London, 1991) p65.

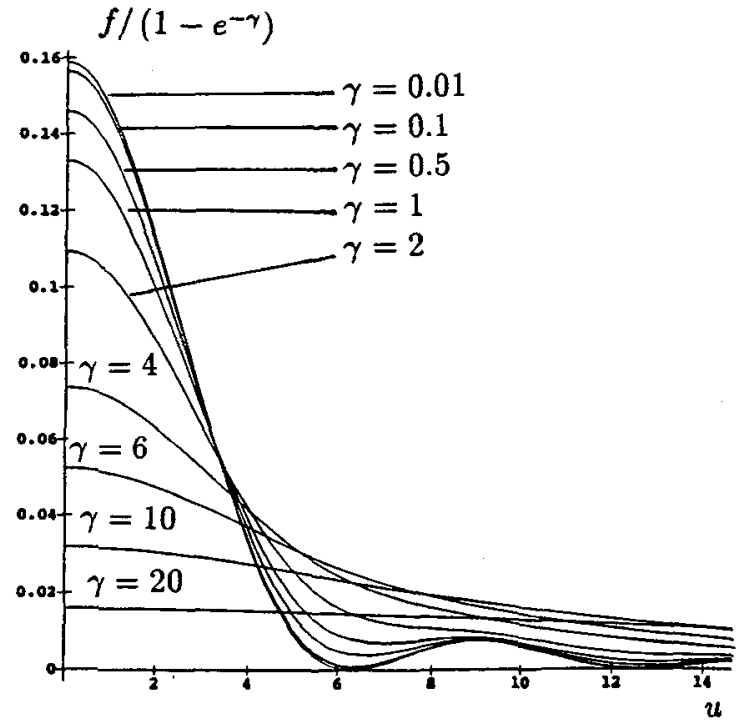

Fig 1 : Détermination de $s_{v}(\varpi)$ à l'aide de (\$)

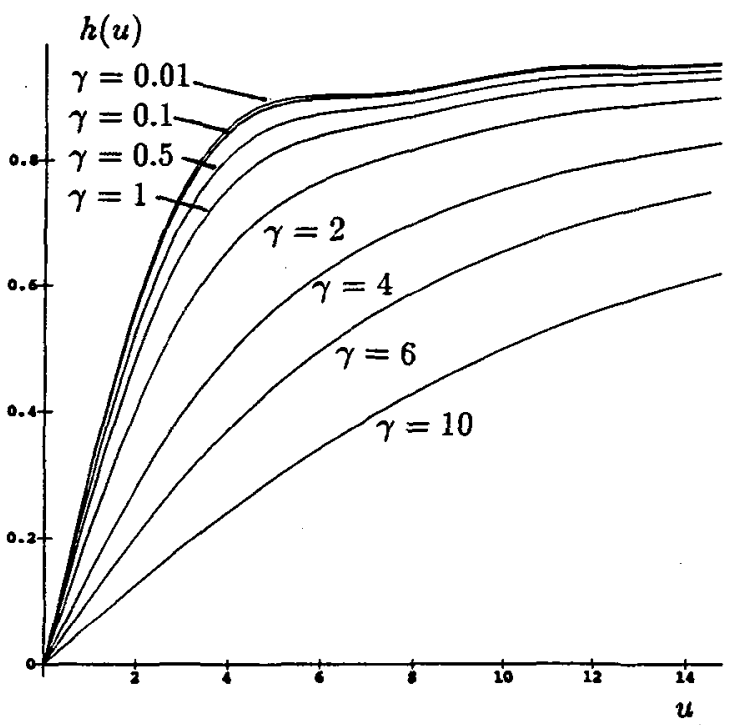

Fig 2 : Détermination de $S_{v}(\varpi)$ à l'aide de (10) 